\author{
МОДЕЛИРОВАНИЕ ДАТЧИКА СИСТЕМЫ \\ ОБНАРУЖЕНИЯ МЕТАЛЛИЧЕСКИХ ЧАСТИЦ \\ В МАСЛОСИСТЕМЕ МАШИН И АГРЕГАТОВ
}

\title{
MODELING OF METALLIC PARTICLES DETECTION SYSTEM SENSOR IN OIL SYSTEM OF MACHINES AND UNITS
}

\author{
М.И. Кокоть, Н.А. Ишинбаев, В.С. Федоров \\ Уфимский государственный нефтяной технический университет, \\ Уфа, Российская Федерация \\ Maksim I. Kokot, Nikolay A. Ishinbaev, Vladimir S. Fedorov \\ Ufa State Petroleum Technological University, Ufa, Russian Federation \\ e-mail: inaufa@yandex.ru
}

Аннотация. Надёжность узлов и механизмов играет ключевую роль при эксплуатации аппаратов, используемых в процессе добычи и переработки нефти. Одним из ключевых аспектов, являющихся причинами снижения работоспособности машин, а также возникновения их отказов, является наличие частиц износа трущихся деталей в смазочном масле.

В данной статье рассматривается моделирование физических процессов, происходящих при попадании неферромагнитных металлических частиц в рабочую область электромагнитного датчика. Произведен анализ физических процессов, возникающих при движении частицы между катушками. В основе данных физических процессов лежит возникновение вихревых токов в частице при попадании в рабочую область датчика. Путем использования результатов данного анализа была получена аналитическая модель выходного сигнала датчика. Полученная модель позволила выявить закономерности изменения амплитуды 
электродвижущей силы (ЭДС), возникающей в измерительной обмотке датчика, от параметров системы. Она связывает между собой ЭДС, геометрические параметры датчика, параметры катушек возбуждения и детектируемой частицы. Так, выходной сигнал прямо пропорционален силе тока, числу витков измерительной обмотки и нелинейно зависит от частоты питающего напряжения и геометрических параметров датчика. Данная зависимость позволила рассчитать амплитуду ЭДС в измерительной обмотке при заданных параметрах датчика и детектируемой частицы. В дальнейшем, имея представление о форме и величине сигнала, можно однозначно определить наличие металлических частиц износа в маслосистеме машин и агрегатов. Результаты данного исследования могут быть использованы для серийного производства датчиков.

Abstract. The reliability of components and mechanisms plays a key role in the operation of devices used in the process of oil production and refining. One of the key aspects that are the reasons for reducing the efficiency of machines, as well as the occurrence of their failures, is the presence of wear particles of rubbing parts in the lubricating oil.

This article discusses the modeling of physical processes that occur when non-ferromagnetic metal particles fall into the working area of an electromagnetic sensor. An analysis of the physical processes that occur when a particle moves between coils. The basis of these physical processes is the occurrence of eddy currents in the particle when it enters the working area of the sensor. Using the results of this analysis, an analytical model of the sensor output signal was obtained. The resulting model allowed to identify patterns of changes in the amplitude of the electromotive force (EMF) that occurs in the measuring winding of the sensor, on the parameters of the system. It connects the EMF, the geometrical parameters of the sensor, the parameters of the excitation coils and the detected particle. Thus, the output signal is directly proportional to the current strength, the number of turns of the measuring 
winding and non-linearly depends on the frequency of the supply voltage and the geometric parameters of the sensor. This dependence made it possible to calculate the amplitude of the EMF in the measuring winding for the given parameters of the sensor and the detected particle. Further, having an idea of the shape and size of the signal, it is possible to unambiguously determine the presence of metallic wear particles in the oil system of machines and units. The results of this study can be used for mass production of sensors.

Ключевые слова: трибодиагностика, система, электромагнитный датчик, катушка, металлическая частица, математическая модель, вихревые токи

Key words: tribodiagnostics, system, electromagnetic sensor, coil, metallic particle, mathematical model, eddy current

Как известно, производственные процессы в нефтегазовой отрасли являются непрерывными, и каждая остановка агрегата несет в себе убыток для компаний. В таких условиях на первый план выходят методы диагностики состояния производственного оборудования, которые позволяют прогнозировать неисправности и отказы агрегатов. Одним из перспективных направлений является трибодиагностика - метод анализа смазочного масла, по наличию и концентрации частиц износа в котором можно сделать вывод о состоянии машин и агрегатов.

Использование трибодиагностики позволяет уменьшить затраты на амортизацию оборудования и обеспечивает безопасность его эксплуатации благодаря предупреждению аварийных и предаварийных состояний, а также повышает безопасность проведения ремонтных и профилактических работ обслуживающего персонала.

На сегодняшний день рынок проточных средств и методов трибодиагностики недостаточно укомплектован. Конечные потребители не могут подобрать подходящие по цене и качеству приборы и продолжают 
использовать устаревшие методы диагностики. Поэтому развитие проточных методов и средств их реализации трибодиагностики является актуальной.

В данной статье проводится моделирование физических процессов, происходящих при попадании неферромагнитных металлических частиц в рабочую область электромагнитного датчика для повышения его метрологических характеристик.

Упрощенная конструкция датчика представлена на рисунке 1. Датчик содержит две катушки возбуждения (правая и левая) и измерительную катушку (средняя). Измерительная катушка выполнена по дифференциальной схеме (катушка с заземленной средней точкой), что обеспечивает эффективное подавление синфазных помех. Катушки возбуждения, по которым протекает переменный ток высокой частоты, являются симметричными и включены таким образом, чтобы их магнитные поля были направлены в противоположных направлениях. Принцип действия датчика основан на явлении электромагнитной индукции - возникновении ЭДС в измерительной катушке при прохождении неферромагнитной металлической частицы. При отсутствии металлических частиц в зоне датчика, a также при нахождении металлической частицы в плоскости измерительной катушки магнитный поток, пронизывающий измерительную катушку, равен нулю из-за симметрии системы [1]. При попадании неферромагнитной металлической частицы в зону датчика, например в область левой катушки, как показано на рисунке 1, симметрия нарушается: в частице возникают вихревые токи под действием переменного магнитного поля, и электромагнитное равновесие системы нарушается. Вихревые токи создают свой магнитный поток, стремящийся, в соответствии с правилом Ленца, ослабить изменение основного потока. При движении металлической частицы до измерительной катушки этот поток уменьшается до нуля. Если рассматривать дальнейшее движение частицы 
до правой катушки, то амплитуда магнитного потока снова возрастает, но сам магнитный поток будет протекать с фазовым сдвигом, равным $\pi$ [2, 3]. Для определения зависимости ЭДС, возникающей в измерительной катушке, от параметров системы рассмотрим движение неферромагнитной металлической частицы через рабочую зону датчика. Введем систему координат с центром в точке, соответствующей центру левой катушке возбуждения, как показано на рисунке 1. Будем считать, что частица движется вдоль оси ОХ.

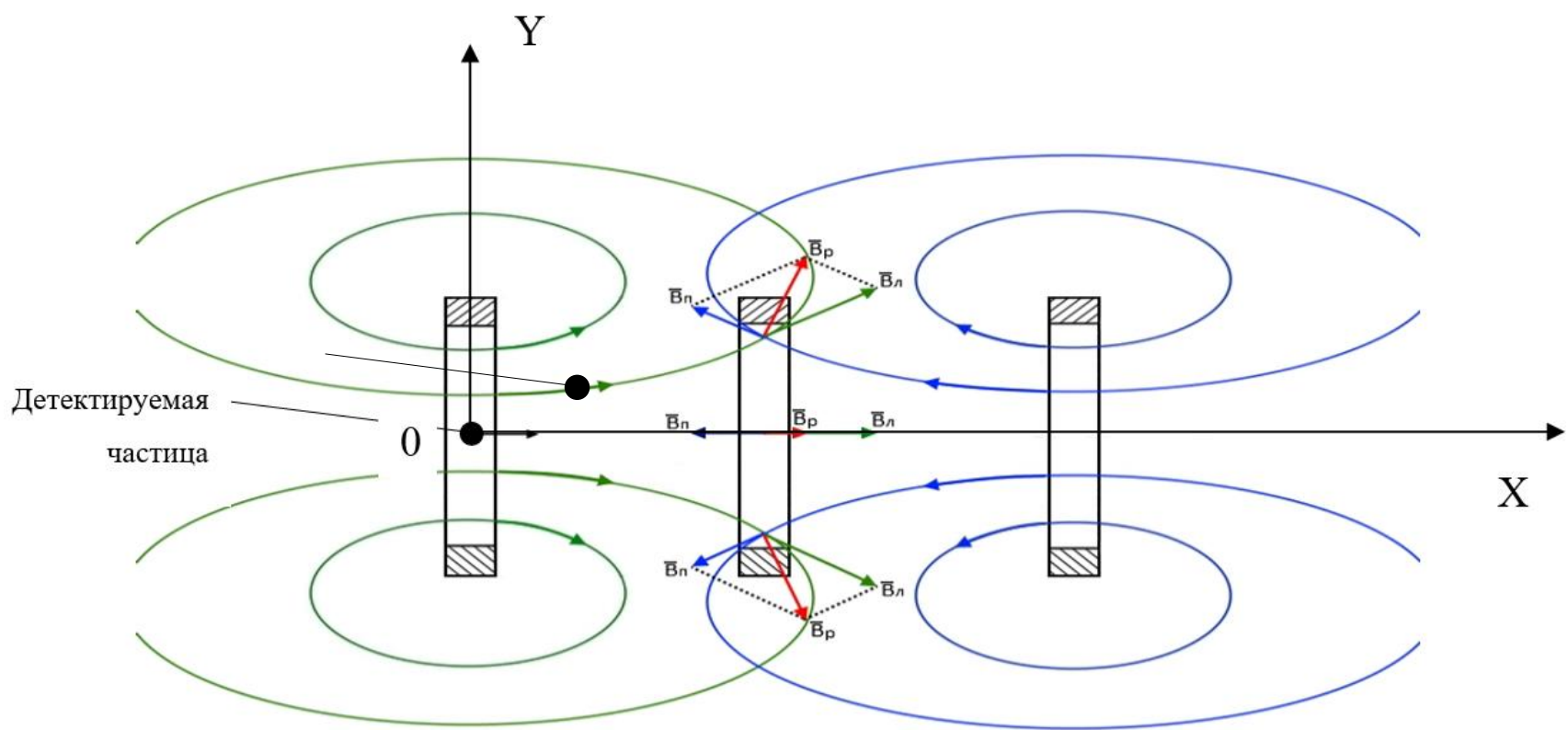

Рисунок 1. Картина магнитных полей при прохождении неферромагнитной металлической частицы через левую катушку

Используя закон Био-Савара-Лапласа и принцип суперпозиции полей, было получено выражение для расчета амплитуды модуля индукции результирующего магнитного поля $B_{\mathrm{p}}$ от двух катушек в исследуемой точке [4-7]:

$$
\begin{aligned}
B_{\mathrm{p}}= & \frac{\mu_{0}}{2} \cdot \frac{I \cdot R^{2} \cdot N}{\left(R^{2}+l^{2}\right)^{\frac{3}{2}}}-\frac{\mu_{0}}{2} \cdot \frac{I \cdot R^{2} \cdot N_{\mathrm{H}}}{\left(R^{2}+(r-l)^{2}\right)^{\frac{3}{2}}}= \\
= & \frac{\mu_{0} \cdot I \cdot R^{2} \cdot N_{\mathrm{H}}}{2} \cdot\left(\left(\frac{1}{R^{2}+l^{2}}\right)^{\frac{3}{2}}-\left(\frac{1}{R^{2}+(r-l)^{2}}\right)^{\frac{3}{2}}\right),
\end{aligned}
$$


где $B_{\mathrm{p}}$ - модуль индукции результирующего магнитного поля от двух катушек в исследуемой точке;

$r$ - расстояние между левой и правой катушками;

I - амплитуда токов, протекающих по намагничивающим катушкам;

$R$ - средние радиусы катушек;

$l$ - координата частицы;

$N_{\text {н }}$ - число витков намагничивающих катушек.

В металлической частице возникают вихревые токи при попадании в переменное магнитное поле катушки (рисунок 2). Для их расчета выделим n = 1000 замкнутых контуров в частице. Для каждого контура с радиусом $x$ найдем амплитуду токов и магнитной индукции, возникающей от них в центре измерительной катушки [8].

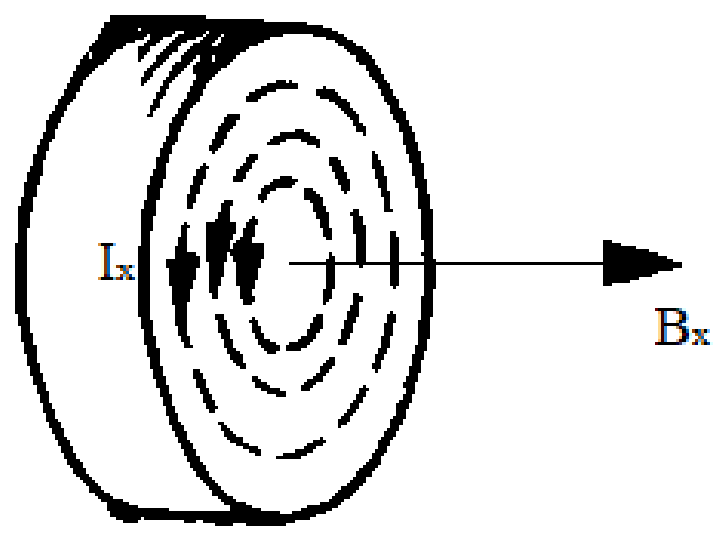

Рисунок 2. Возникновение вихревых токов в металлической частице

Для дальнейших расчетов примем, что частица имеет форму цилиндрической пластины [8].

Амплитуда ЭДС, возникающей в контуре $x$, определяется по формуле:

$$
E_{x}=\frac{d \Phi}{d t}=B_{\mathrm{p}} \cdot S_{\mathrm{K}} \cdot 2 \cdot \pi \cdot f=B_{\mathrm{p}} \cdot 2 \cdot \pi^{2} \cdot x^{2} \cdot f,
$$

где $E_{x}$ - амплитуда ЭДС, возникающей в контуре $x$;

$S_{\mathrm{K}}-$ площадь контура $x$;

$f$ - частота тока в катушках возбуждения;

$x$ - радиус контура. 
Индуктивное сопротивление контура $x$ определяется по формуле

$$
X_{l ч}=2 \cdot \pi \cdot f \cdot L,
$$

где $X_{l ч}-$ индуктивное сопротивление контура, Ом;

$L-$ индуктивность контур $x$, Гн.

Индуктивность контура $x$ определяется по формуле [9]:

$$
L=0,0004 \cdot \pi \cdot x \cdot\left(\ln \left(\frac{16 \cdot x}{d_{\mathrm{K}}}\right)-1,75\right),
$$

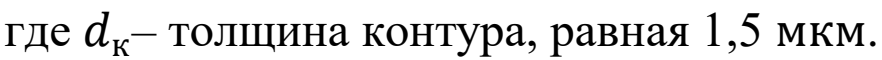

Подставив выражение (4) в (3), получим:

$$
X_{l \mathrm{\Psi}}=0,0008 \cdot \pi^{2} \cdot f \cdot x \cdot\left(\ln \left(\frac{16 \cdot x}{d_{\mathrm{K}}}\right)-1,75\right) .
$$

Полное сопротивление контура $x$ определяется по формуле:

$$
\begin{gathered}
Z=\sqrt{R_{x}^{2}+X_{l \mathrm{u}}^{2}}= \\
=2 \cdot \pi \cdot x \cdot \sqrt{\left(\frac{\rho}{l_{\mathrm{u}} \cdot d_{\mathrm{K}}}\right)^{2}+0,0004^{2} \cdot \pi^{2} \cdot f^{2} \cdot\left(\ln \left(\frac{16 \cdot x}{d_{\mathrm{K}}}\right)-1,75\right)^{2}},
\end{gathered}
$$

где $Z$ - полное сопротивление контура $x$;

$R_{x}$ - активное сопротивление контура $x$;

$\rho$ - удельное сопротивление контура, равное $10^{-7} \mathrm{OM} \cdot \mathrm{M}$;

$l_{\text {ч }}$ - длина частицы.

Амплитуда тока в контуре $x$ определяется по формуле

$$
\begin{gathered}
I_{x}=\frac{E_{x}}{Z}=\frac{B_{\mathrm{p}} \cdot 2 \cdot \pi^{2} \cdot f \cdot x^{2}}{2 \cdot \pi \cdot x \cdot \sqrt{\left(\frac{\rho}{l_{\mathrm{u}} \cdot d_{\mathrm{K}}}\right)^{2}+0,0004^{2} \cdot \pi^{2} \cdot f^{2} \cdot\left(\ln \left(\frac{16 \cdot x}{d_{\mathrm{K}}}\right)-1,75\right)^{2}}} \\
=\frac{B_{\mathrm{p}} \cdot \pi \cdot f \cdot x}{\sqrt{\left(\frac{\rho}{l_{\mathrm{q}} \cdot d_{\mathrm{K}}}\right)^{2}+0,0004^{2} \cdot \pi^{2} \cdot f^{2} \cdot\left(\ln \left(\frac{16 \cdot x}{d_{\mathrm{K}}}\right)-1,75\right)^{2}}}
\end{gathered}
$$

где $\quad I_{x}-$ амплитуда тока в контуре $x, \mathrm{~A}$. 
Амплитуда магнитной индукции в середине измерительной катушки, создаваемая током в контуре $x$, определяется по формуле:

$$
\begin{gathered}
B_{x}=\frac{\mu_{0}}{2} \cdot \frac{I_{x} \cdot x^{2}}{\left(x^{2}+\left(r_{13}-l\right)^{2}\right)^{\frac{3}{2}}}= \\
=\frac{\mu_{0} \cdot B_{\mathrm{p}} \cdot \pi \cdot f \cdot x^{3}}{2 \cdot\left(x^{2}+\left(r_{13}-l\right)^{2}\right)^{\frac{3}{2}} \cdot \sqrt{\left(\frac{\rho}{l_{\mathrm{Y}} \cdot d_{\mathrm{K}}}\right)^{2}+0,0004^{2} \cdot \pi^{2} \cdot f^{2} \cdot\left(\ln \left(\frac{16 \cdot x}{d_{\mathrm{K}}}\right)-1,75\right)^{2}}},
\end{gathered}
$$

где $B_{x}$ - амплитуда магнитной индукции в середине измерительной катушки, создаваемая током в контуре $x$, Тл.

Используя принцип суперпозиции магнитных полей, найдем амплитуду магнитной индукции в середине измерительной катушки, создаваемой вихревыми токами частицы:

$$
\begin{gathered}
B=\frac{\mu_{0}}{2} \cdot \frac{I_{x} \cdot x^{2}}{\left(x^{2}+\left(r_{13}-l\right)^{2}\right)^{\frac{3}{2}}}= \\
=\frac{\mu_{0} \cdot B_{\mathrm{p}} \cdot \pi \cdot f}{2} \times \\
\times \sum_{i=1}^{n-1} \frac{\left(i \cdot d_{\mathrm{K}}\right)^{3}}{\left(\left(i \cdot d_{\mathrm{K}}\right)^{2}+\left(r_{13}-l\right)^{2}\right)^{\frac{3}{2}} \cdot \sqrt{\left(\frac{\rho}{l_{\mathrm{l}} \cdot d_{\mathrm{K}}}\right)^{2}+0,0004^{2} \cdot \pi^{2} \cdot f^{2} \cdot\left(\ln \left(\frac{16 \cdot i \cdot d_{\mathrm{K}}}{d_{\mathrm{K}}}\right)-1,75\right)^{2}}}= \\
=\frac{\mu_{0} \cdot B_{\mathrm{p}} \cdot \pi \cdot f}{2} \cdot \sum_{i=1}^{n-1} \frac{\left(i \cdot d_{\mathrm{K}}\right)^{3}}{\left(\left(i \cdot d_{\mathrm{K}}\right)^{2}+\left(r_{13}-l\right)^{2}\right)^{\frac{3}{2}} \cdot \sqrt{\left(\frac{\rho}{l_{\mathrm{q}} \cdot d_{\mathrm{K}}}\right)^{2}+0,0004^{2} \cdot \pi^{2} \cdot f^{2} \cdot(\ln (16 \cdot i)-1,75)^{2}}}
\end{gathered}
$$

где $B$ - амплитуда магнитной индукции в середине измерительной катушки, создаваемая вихревыми токами частицы;

$n$ - число замкнутых контуров в частице.

Амплитуда ЭДС, возникающей в измерительной обмотке при движении частицы через рабочую зону датчика, находится по формуле:

$$
\begin{gathered}
E=N \cdot \frac{d \Phi}{d t}=-N \cdot B \cdot S \cdot 2 \cdot \pi \cdot f=-\frac{N \cdot \mu_{0}^{2} \cdot \pi^{3} \cdot f^{2} \cdot R^{4} \cdot I \cdot N_{\mathrm{H}}}{2} \cdot\left(\left(\frac{1}{R^{2}+l^{2}}\right)^{\frac{3}{2}}-\right. \\
\left.-\left(\frac{1}{R^{2}+(r-l)^{2}}\right)^{\frac{3}{2}}\right) \cdot \sum_{i=1}^{n-1} \frac{\left(i \cdot d_{\mathrm{K}}\right)^{3}}{\left(\left(i \cdot d_{\mathrm{K}}\right)^{2}+\left(r_{13}-l\right)^{2}\right)^{\frac{3}{2}} \cdot \sqrt{\left(\frac{\rho}{l_{\mathrm{l}} \cdot d_{\mathrm{K}}}\right)^{2}+0,0004^{2} \cdot \pi^{2} \cdot f^{2} \cdot(\ln (16 \cdot i)-1,75)^{2}}}
\end{gathered}
$$


где $E$ - амплитуда ЭДС, возникающей в измерительной обмотке при движении частицы через рабочую зону датчика;

$N$ - число витков в измерительной катушке;

$S$ - площадь контура измерительной катушки.

Полученная зависимость связывает между собой амплитуду ЭДС в измерительной катушке с геометрическими $\left(R, r, r_{13}\right)$ параметрами датчика, параметрами катушек $\left(N, N_{\mathrm{H}}, I\right)$ и детектируемой частицы $\left(\rho, l_{\mathrm{q}}\right.$, $\left.d_{\kappa}, l\right)$. Построим график зависимости амплитуды ЭДС в измерительной катушке от координаты частицы (рисунок 3).

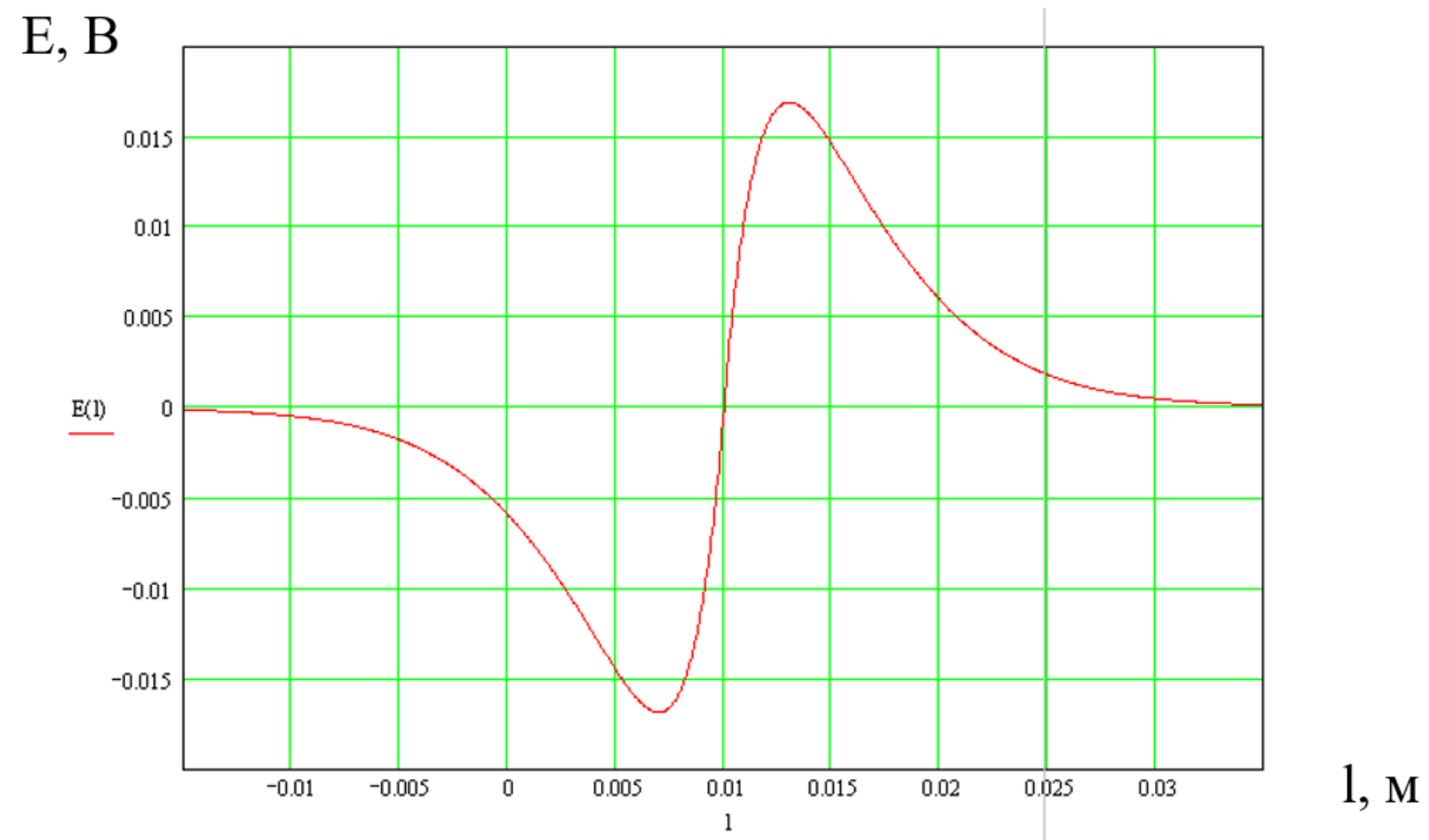

Рисунок 3. График зависимости амплитуды ЭДС в измерительной катушке от координаты частицы

Количество витков в каждой из намагничивающих катушек равно 16 шт., а в измерительной - 40 шт. Сила тока в витках намагничивающих катушек 1 и 2 равна 0,16 A, частота тока равна 100 кГц. Радиус катушек равен 0,0125 м. Для расчета принимаем намагничивающие катушки как одновитковые, по которым протекает ток амплитудой 2,5 А. Радиус частицы равен 1,5 мм, а ее длина 0,15 мм.

Полученный график имеет экстремум, равный 17 мВ. 


\section{Выводы}

Полученная аналитическая модель (10) позволяет решать следующие задачи:

1. Установить связь между амплитудой ЭДС и геометрическими параметрами датчика, параметрами катушек и детектируемой частицы. Так, выходной сигнал прямопропорционален силе тока, числу витков измерительной обмотки и нелинейно зависит от частоты питающего напряжения и геометрических параметров датчика;

2. Произвести расчет амплитудного значения ЭДС при заданных параметрах электромагнитного датчика и детектируемой частицы;

3. Однозначно определять наличие неферромагнитных металлических частиц износа в маслосистеме машин и агрегатов.

\section{Список использованных источников}

1. Трофимова Т.И. Курс физики. М.: Академия, 2006. 560 с.

2. Савельев И.В. Курс общей физики. М.: Наука, 1982. Т. 2 «Электричество и магнетизм. Волны. Оптика». 496 с.

3. Тюшев А.Н. Курс лекций по физике. Новосибирск: СГГА, 2003. Ч. 2: «Электричество и магнетизм». $150 \mathrm{c}$.

4. Применение закона Био-Савара-Лапласа для нахождения магнитного поля. URL: http://lektsia.com/2x14d7.html (дата обращения: 20.11.2018).

5. Магнитное поле на оси кругового тока. URL: http://online.mephi.ru/courses/physics/electricity/data/course/6/6.3.html (дата обращения: 20.11.2018).

6. Fan H. Experimental Study of an On-Line Monitoring Sensor for Wear Particles in Oil // Tribology. 2010. No. 30 (4). P. 338-343.

7. Lee H.-B. Efficient Magnetic Field Calculation Method for Pancake Coil Using Biotsavart Law // 12th Biennial IEEE Conference on Electromagnetic Field Computatio. 2006. 193 p. 
8. Теоретические основы электротехники и электроники. URL: http://bourabai.kz/toe/chapter03.htm (дата обращения: 27.11.2018).

9. Расчет индуктивности одиночного круглого витка. URL: http://coil32.ru/man/loop.html (дата обращения: 20.11.2018).

\section{References}

1. Trofimova T.I. Kurs fiziki [Physics Course]. Moscow, Academy, 2006. 560 p. [in Russian].

2. Savel'ev I.V. Kurs obshchei fiziki. T. 2. Elektrichestvo i magnetizm. Volny. Optika. [General Physics Course. Vol. 2. Electricity and Magnetism. Waves. Optics]. Moscow, Nauka, 1982. 496. [in Russian].

3. Tyushev A.N. Kurs lektsii po fizike. Chast' 2. Elektrichestvo i magnetizm [A Course of Lectures in Physics. Part 2. Electricity and Magnetism.]. Novosibirsk, SSUGT, 2003. 150 p. [in Russian].

4. Primenenie zakona Bio-Savara-Laplasa dlya nakhozhdeniya magnitnogo polya [The Application of the Law of Biot-Savart-Laplace's Law to Find Magnetic Field]. Available at: http://lektsia.com/2x14d7.html (accessed 20.11.2018). [in Russian].

5. Magnitnoe pole na osi krugovogo toka [The Magnetic Field on the Axis of a Circular Current]. Available at: http://online.mephi.ru/courses/ physics/electricity/data/course/6/6.3.html (accessed 20.11.2018). [in Russian].

6. Fan H. Experimental Study of an On-Line Monitoring Sensor for Wear Particles in Oil. Tribology, 2010, No. 30 (4), pp. 338-343.

7. Lee H.-B. Efficient Magnetic Field Calculation Method for Pancake Coil Using BiotSavart Law. 12th Biennial IEEE Conference on Electromagnetic Field Computatio. 2006. 193 p.

8. Teoreticheskie osnovy elektrotekhniki i elektroniki [Theoretical Bases of Electrical Engineering and Electronics]. Available at: http://bourabai.kz/toe/chapter03.htm (accessed 27.11.2018). [in Russian]. 
9. Raschet induktivnosti odinochnogo kruglogo vitka [Calculation of Inductance of a Single Round Coil]. Available at: http://coil32.ru/man/loop.html (accessed 20.11.2018). [in Russian].

\section{Сведения об авторах}

\section{About the authors}

Кокоть Максим Игоревич, магистрант кафедры «Автоматизация технологических процессов и производств», УГНТУ, г. Уфа, Российская Федерация

Maksim I. Kokot, Undergraduate Student of Automation of Technological Processes and Industrial Facilities Department, USPTU, Ufa, Russian Federation

e-mail: max.kokot@yandex.ru

Ишинбаев Николай Александрович, канд. техн. наук, доцент кафедры «Автоматизация технологических процессов и производств», УГНТУ, г. Уфа, Российская Федерация

Nikolay A. Ishinbaev, Candidate of Engineering Sciences, Assistant Professor of Automation of Technological Processes and Industrial Facilities Department, USPTU, Ufa, Russian Federation

e-mail: inaufa@yandex.ru

Федоров Владимир Сергеевич, магистрант кафедры «Автоматизация технологических процессов и производств», УГНТУ, г. Уфа, Российская Федерация

Vladimir S. Fedorov, Undergraduate Student of Automation of Technological Processes and Industrial Facilities Department, USPTU, Ufa, Russian Federation

e-mail: vladimirfedorovs@mail.ru 